\title{
Peculiarities of diagnostics of billiary hypertension in patients with complicated forms of chronic pancreatitis
}

\author{
Volodymyr Pylypchuk \\ Department of Surgery and Cardiac Surgery, Ivano-Frankivsk National Medical University, Ivano-Frankivsk, Ukraine
}

Gastroenterology Rev 2018; 13 (2): 143-149

DOI: https://doi.org/10.5114/pg.2018.75823

Key words: chronic pancreatitis, biliary hypertension, biliary pressure, mechanical jaundice.

Address for correspondence: Assoc. Prof. Volodymyr Pylypchuk MD, PhD, Department of Surgery and Cardiac Surgery, Ivano-Frankivsk National Medical University, 23 Konovaltsia St, 76018 Ivano-Frankivsk, Ukraine, phone: +38 0990454538, e-mail: vipdoz@ukr.net

\begin{abstract}
Introduction: The morbidity of chronic pancreatitis (CP) remains at a high level. In cases of CP, prepapillary stenosis of the common bile duct (CBD) complicates the course of disease in 30-60\% of patients.

Aim: To improve the detection of biliary hypertension $(\mathrm{BH})$ in patients with complicated forms of $\mathrm{CP}$ by increasing the accuracy of preoperative and intraoperative diagnostics using modern diagnostic methods.

Material and methods: We analysed the results of surgical treatment of 573 patients with complicated forms of CP. In 163 (28.5\%) patients, CP was complicated by BH. The method of intraoperative monitoring of biliary pressure (IOM BP) was developed and introduced for intraoperative control and determination of the adequacy of intervention regarding biliary decompression.

Results: Mechanical jaundice was diagnosed by clinical methods in 101 (61.9\%) CP patients with BH, and by laboratory methods in 108 (66.2\%) patients. Such methods as magnetic resonance cholangiopancreatography (95.6\%), endoscopic retrograde cholangiopancreatography (93.7\%), and computed tomography $(93.8 \%)$ proved to have the highest sensitivity for the diagnostics of $\mathrm{BH}$ in cases of $\mathrm{CP}$.

Conclusions: The application of an integrated approach with extensive use of modern non-invasive preoperative methods of assessment allowed an approximation of the reliability of preoperative diagnostics of BH in CP patients to $95.6 \%$. The use of the IOM BP method enables us to increase the sensitivity of intraoperative diagnostics of BH to $97.3 \%$ and choose the method of surgical intervention, which significantly reduces the risk of $\mathrm{BH}$ recurrence in the distant postoperative period by $15.1 \%$ $\left(\chi^{2}=4.22, p=0.04\right)$.
\end{abstract}

\section{Introduction}

The morbidity of chronic pancreatitis (CP) in the world is 3.1-8 cases, and in European countries it is 4-8 cases. Its prevalence amounts to 25 cases per 100,000 people; in 2012 the morbidity rate of pancreas pathology in Ukraine amounted to 226 cases per 100,000 people, and its prevalence constituted 2471 cases per 100,000 people [1]. Every year, the number of patients with pancreas pathology in Ukraine increases by 5000 6000 [2]. The number of patients with CP has doubled over the past 30 years $[3,4]$.

In cases of $\mathrm{CP}$, pre-papillary stenosis of the common bile duct (CBD) complicates the course of the disease in $30-60 \%$ of patients [5-8]; biliary hypertension $(\mathrm{BH})$ is manifested by obstructive jaundice in $25-55 \%$ of pa- tients. In $57 \%$ of cases, it is diagnosed by instrumental methods $[9,10]$. In addition to tubular stenosis caused by pancreatic fibrosis and inflammation of the head of the pancreas $[7,11]$, the causes of CBD obstruction in cases of CP may include pseudocysts, occasional exacerbation of the inflammatory process in the head of the pancreas, stenosis of the major duodenal papilla, and compression of the terminal end of CBD by inflammatory masses [11].

Regarding the remote postoperative period after surgeries for $\mathrm{CP}, \mathrm{BH}$ manifests itself in $25 \%$ of patients who underwent draining surgeries and $8-9 \%$ of patients who underwent resection surgeries [12].

Currently, there is no clear consensus on the optimal diagnostic method and the use of different examination methods in cases of CP $[13,14]$, let alone CP with signs 
of $\mathrm{BH}$. Diagnostics of $\mathrm{CP}$ with $\mathrm{BH}$ is based on the assessment of structural and functional changes in the pancreas and adjoining organs, in particular the bile ducts and the liver. Ultrasound examination, computed tomography $(\mathrm{CT})$, endoscopic retrograde cholangiopancreatography (ERCP), and magnetic resonance cholangiopancreatography $(\mathrm{MRCP})$ remain the most informative methods of preoperative diagnostics of $\mathrm{BH}$ in $\mathrm{CP}$ patients.

\section{Aim}

The objective of the work is to improve the detection of $\mathrm{BH}$ in patients with complicated forms of CP by increasing the accuracy of preoperative and intraoperative diagnostics using modern diagnostic methods.

\section{Material and methods}

We analysed the results of examinations and treatment of 573 patients with CP, 163 (28.4\%) of whom had symptoms of $\mathrm{BH}$. Patients with $\mathrm{CP}$ and $\mathrm{BH}$ were divided into two groups: group 1 (main group) included 36 (22.1\%) patients in whom $\mathrm{BH}$ was diagnosed using the methods proposed by us, in particular, intraoperative monitoring of biliary pressure (IOM BP), as well as the developed diagnostic and treatment schemes (prospective period 2014-2016); group 2 (comparison group) included 127 (77.9\%) patients in whom $\mathrm{BH}$ was diagnosed by the generally accepted methods (retrospective period 2009-2013). The groups are indiscriminate and comparable in terms of patients' age, sex, and the course of the disease $(p>0.05)$.

The number of men significantly exceeded the number of women in both groups: there were 9 (5.5\%) women and 154 (94.5\%) men aged 21-63 years, and mean age $45.8 \pm 0.8$ years.

The distribution by age in the first group was as follows: $30-39$ years old - 10 (27.8\%) patients, 40-49 years old $-13(36.1 \%)$ patients, 50-59 years old 12 (33.3\%) patients, and 60 years and older - 1 (2.8\%) patient. The distribution of patients by age in the sec- ond group was as follows: under $30-7$ (5.5\%) patients, 30-39- 42 (33.0\%) patients, 40-49- 33 (26.0\%) patients, 50-59- 37 (29.3\%) patients, and 60 and over $8(6.3 \%)$ patients.

The following methods were used to diagnose $\mathrm{BH}$ : laboratory examinations (levels of total and conjugated bilirubin, and alkaline phosphatase), ultrasonography (USG), ERCP, CT, and MRCP.

The method of IOM BP was proposed and applied in patients of the main group $[15,16]$. It was done for diagnostic (intraoperative detection of $\mathrm{BH}$, which was not diagnosed at the preoperative stage) and tactical (correction of surgical intervention to eliminate $\mathrm{BH}$ ) reasons.

Three degrees of $\mathrm{BH}$ were differentiated: $\mathrm{BH}$ of the $1^{\text {st }}$ degree - pressure in the CBD increased from 160 to $190 \mathrm{~mm} \mathrm{WG}$; $\mathrm{BH}$ of the $2^{\text {nd }}$ degree - increase of the biliary pressure from 190 to $250 \mathrm{~mm}$ WG; $\mathrm{BH}$ of the $3^{\text {rd }}$ degree - pressure in ducts invariably exceeds $250 \mathrm{~mm} \mathrm{WG}$.

\section{Results and discussion}

While studying the aetiological factors of CP with $\mathrm{BH}$, it was noted that 67 (41.1\%) CP patients with $\mathrm{BH}$ indicated long-term and systematic tobacco smoking. In 44 (26.9\%) patients, a direct connection was established between CP and regular alcohol consumption. Frequent consumption of alcohol combined with tobacco smoking was seen in 28 (82.3\%) patients. In 32 (19.6\%) patients, we attributed the development of $\mathrm{CP}$ with earlier acute pancreatitis in their medical history and the transition from acute to chronic pancreatitis. Another 9 (5.5\%) patients had a biliary cause of CP (they underwent surgeries for cholelithiasis).

In $11(6.7 \%)$ patients we failed to establish a clear connection between the development of $\mathrm{CP}$ with $\mathrm{BH}$ and any cause. We found neither hereditary factors nor connections with consumption of fatty food or other factors. Thus, CP was characterised as idiopathic in their cases (Table I).

Table I. Distribution of CP patients with $\mathrm{BH}$ according to aetiological factors

\begin{tabular}{|c|c|c|c|c|c|}
\hline \multirow[t]{3}{*}{ CP aetiology } & \multicolumn{4}{|c|}{ Patient groups } & \multirow[t]{3}{*}{$P$-value } \\
\hline & \multicolumn{2}{|c|}{ First group $(n=36)$} & \multicolumn{2}{|c|}{ Second group $(n=127)$} & \\
\hline & Mean & $\%$ & Mean & $\%$ & \\
\hline CP of alcoholic origin & 11 & 30.5 & 33 & 25.9 & $>0.05$ \\
\hline Tobacco smoking & 17 & 47.2 & 50 & 39.4 & $>0.05$ \\
\hline CP after acute pancreatitis & 6 & 16.6 & 26 & 20.5 & $>0.05$ \\
\hline CP of biliary origin & 1 & 2.7 & 8 & 6.3 & $>0.05$ \\
\hline Idiopathic CP & 1 & 2.7 & 10 & 7.8 & $>0.05$ \\
\hline
\end{tabular}


One hundred and eleven (68.1\%) patients were earlier treated for acute or chronic pancreatitis (some of them repeatedly) in other gastroenterological and surgical inpatient facilities. Another 26 (15.9\%) patients received outpatient treatment only, and 26 (15.9\%) patients never consulted doctors regarding problems with their pancreases.

Surgical treatment of the diseases of the pancreas was previously administered in 35 (21.5\%) patients: 9 (25.0\%) patients of the first group and 26 (20.5\%) patients of the second group. Surgeries were performed on 9 (5.5\%) patients suffering from acute destructive pancreatitis and pancreatic necrosis: 1 (2.8\%) patient of the first group and $8(6.3 \%)$ patients of the second group.

Twenty-six (15.9\%) patients were previously surgically treated for CP: 8 (22.2\%) patients of the first group and 18 (14.2\%) patients of the second group. They underwent the following interventions:

- ERCP with endobiliary stent placement - 7 (4.3\%) patients;

- ERCP with stent placement into the Wirsung duct 3 (1.8\%) patients;

- Frey's procedure - 2 (1.2\%) patients;

- lateral pancreatic enterostomy (LPES) - 6 (3.9\%) patients;

- cystoenterostomy - 2 (1.2\%) patients;

- external drainage of cysts of the pancreas - 5 (3.1\%) patients;

- hepatic enteroanastomosis (HEA) - 1 (0.6\%) patient.

Clinical manifestations of the disease were non-specific, but a pain syndrome and signs of exocrine insufficiency were noted in the majority of patients. Clinical examination of $\mathrm{CP}$ patients with $\mathrm{BH}$ indicated severe impairment of different organs and systems. The duration of clinical manifestations of $\mathrm{CP}$ with $\mathrm{BH}$ in both groups ranged from 6 months to 10 years, on average $5.2 \pm 1.2$ years.

Pain syndrome was noted in 138 (84.6\%) patients. In 111 (80.4\%) patients, the pain was persistent (type A) and increased after meals; in 27 (19.6\%) patients it was transient and spasmodic (type B). In 121 (87.7\%) patients the pain was localised in the epigastric region, in $12(8.7 \%)$ - in the left hypochondrium, and in 5 (3.6\%) patients - in the right one. Painkillers (including potent ones) were continuously used by 88 (63.7\%) patients.

Fibrous-degenerative changes in the pancreatic tissue with the involvement of nerve endings of the gland into the pathological process were considered the main cause of the pain syndrome in patients with the CP of the pancreatic head. In this case, resection surgeries (Beger procedure, Berne modification) to eliminate the abnormal focus are pathogenetically grounded.
Pancreatic hypertension is another cause of pain. Increased pressure in pancreatic ducts due to their obstruction and restriction of pancreatic juice outflow may cause a severe pain syndrome. In such cases, surgical intervention should aim for pancreatic decompression; drainage operations (lateral pancreatic enterostomy (LPES), cystenterostomy (CET), CPES, and endoscopic stent placement in Wirsung's duct) are very effective.

If the pain syndrome is caused by a combination of both fibrous-degenerative changes in the pancreas and pancreatic hypertension, resection and draining surgery, which can simultaneously eliminate both causes of pain (Frey's procedure), is effective.

Jaundice was diagnosed in 101 (61.9\%) patients: in $20(55.5 \%)$ patients of the first group and in $81(63.7 \%)$ patients of the second group. In 26 (25.7\%) patients, jaundice was recurrent, and in $75(74.3 \%)$ patients it appeared for the first time. In 62 (38.1\%) patients, BH had a subclinical course.

Twenty-seven $(75.0 \%)$ patients of the first group and $91(71.6 \%)$ patients of the second group showed weight loss. Furthermore, 22 (13.5\%) patients lost from 5 to $10 \mathrm{~kg}$ of body weight during the illness, and 14 (8.6\%) patients - more than $10 \mathrm{~kg}$. Dyskinetic syndrome (diarrhoea, constipation) occurred in $68(41.2 \%)$ patients. Dyspeptic syndrome (changes in appetite, nausea, vomiting that does not bring relief, aversion to oily food, flatulence) was noticed in all patients. Such manifestations of the disease prompted $142(87.1 \%)$ patients to take enzyme preparations regularly or occasionally. A pronounced asthenic-vegetative syndrome (general weakness, irritability, sleep disturbance, decreased ability to work) was characteristic of 135 (82.8\%) patients.

During the laboratory examination, hyperbilirubinaemia was detected in 108 (66.2\%) patients; in 55 (33.8\%) patients, $\mathrm{BH}$ was without hyperbilirubinaemia.

As a screening method, USG was performed in all 163 patients. A distention of the suprapancreatic part of the CBD from $8 \mathrm{~mm}$ was considered a specific ultrasound sign of the $\mathrm{BH}$ in cases of CP. Dilatation of bile ducts was diagnosed in 122 (74.8\%) patients. During ultrasound examination, cystic formations were found in the area of the head of the pancreas in 16 (9.8\%) patients; in 3 patients, the cysts were located parapancreatically retroduodenally. However, the distention of the CBD occurred in 11 (68.7\%) of them. Ultrasound examination was used to assess the dynamics of the pathological process and as a criterion for $\mathrm{BH}$ elimination in the postoperative period and before hospital discharge. The distention of CBD (from 8 to $10 \mathrm{~mm}$ ) after surgery persisted in $13.4 \%$ of patients of the second versus $5.6 \%$ of patients of the first group. However, 
there was no significant difference between the groups $(p>0.05)$.

Endoscopic retrograde cholangiopancreatography was used in 32 (19.6\%) patients. In 2 patients, the performance of ERCP was not possible because of technical difficulties due to severe deformity of the duodenum (1 patient) and the presence of a peripapillary duodenal diverticulum (1 patient). In the course of ERCP, the following were considered to be pathognomonic signs of $\mathrm{BH}$ in cases of CP: deformation and stenosis of the major duodenal papilla, tubular stenosis of the intrapancreatic part of the CBD and its suprastenotic expansion, absence of a free passage of contrast substance in the duodenum at the beginning of the examination, and delayed evacuation of the contrast agent (more than 45 min). Expansion of the supra-pancreatic part of the CBD during ERCP was detected in all patients. In 22 (73.3\%) patients, the diameter of the duct exceeded $10 \mathrm{~mm}$, and in seven patients it was larger than $16 \mathrm{~mm}$. The length of tubular stenosis of the intrapancreatic part of CBD during ERCP was under $20 \mathrm{~mm}$ in 2 (6.6\%) patients, $20-26 \mathrm{~mm}$ - in 22 (73.3\%) patients, and over $26 \mathrm{~mm}$ - in $6(20.1 \%)$ patients. An average length of stenosis was $24 \pm 4 \mathrm{~mm}$.

For therapeutic purposes, ERCP was used for endobiliary stent placement in three patients with obstructive jaundice and for papillotomy and choledocho-lithoextraction - in 2 patients. Performance of ERCP in conjunction with papillosphincterotomy and extraction of concretions was considered as an independent surgical intervention, which allowed the removal of obstructive jaundice and cholangitis. Due to the invasiveness of the method and the availability of MRCP in the clinic, the indications for ERCP use were narrowed down. As a diagnostic method, ERCP was used to study the pathology of the major duodenal papilla.

Computed tomography was performed in 113 (69.3\%) patients: 28 (77.7\%) patients in group 1 and $85(66.9 \%)$ in group 2. Biliary hypertension was determined on the basis of distention of the CBD $(8 \mathrm{~mm}$ in diameter and more), presence of pathology in the CBD, expansion of intrahepatic bile ducts, enlargement of the gallbladder, induration of its walls, and the presence of choledochal stenosis in the intrapancreatic part with suprastenotic enlargement. Cysts in the area of the head of the pancreas were diagnosed in 11 patients during CT. Supra-pancreatic enlargement of the bile ducts was diagnosed in 106 (93.8\%) patients.

Magnetic resonance cholangiopancreatography was used in 23 (14.1\%) patients. Unlike ERCP, MRCP allowed visualisation of the bile ducts above and below the level of obstruction and gave a real picture of the state of bile ducts compared with ERCP, in which the introduction of the contrast agent artificially increased duct expansion. Magnetic resonance cholangiopancreatography allowed for precise determination of the level, extent of constrictions and their cause, as well as supra-stenotic extension of the CBD, which was of great practical importance for the choice of surgical approach. Obstruction of the CBD during the examination had a conical appearance; the visualisation of its altered part was traced throughout the pancreatic head. In all cases, supra-stenotic extension of CBD was observed. Tubular stenosis of the intrapancreatic part of the CBD was detected in 21 patients; a stent, which was placed earlier during ERCP for obstructive jaundice, was contrasted in one patient in the lumen of the extrahepatic bile ducts. In one patient, MRCP was less informative.

Intraoperative measurement of the diameter of the bile ducts was performed in 147 (90.2\%) patients during open surgeries. Dilatation of the CBD was found in all patients. In three patients, the examination could not be done because of the marked inflammatory changes in the area of hepatoduodenal ligament.

IOM BP was used in 36 patients. In one more patient, the examination could not be done due to the presence of manifest inflammatory changes in the area of the hepatoduodenal ligament. Performance of IOM $\mathrm{BP}$ at the beginning of surgical intervention allowed diagnosis of $\mathrm{BH}$ of the first degree in $6(16.6 \%)$ patients, $\mathrm{BH}$ of the second degree in 22 (61.1\%) patients, and $\mathrm{BH}$ of the third degree in $8(22.2 \%)$ patients.

Clinical manifestations of $\mathrm{BH}$ occurred in 8 (100.0\%) patients with $\mathrm{BH}$ of the third degree and in 12 (54.5\%) patients with $\mathrm{BH}$ of the second degree. In all patients with $\mathrm{BH}$ of the first degree and in 10 (45.5\%) patients with $\mathrm{BH}$ of the second degree, hypertension had no clinical manifestations and was diagnosed only with the help of instrumental methods.

Reduction of biliary pressure to normal parameters during operation was recorded in 22 (61.1\%) patients who had no jaundice or hyperbilirubinaemia or in whom they were slightly pronounced. They underwent surgical interventions of the pancreas without intervention on bile ducts. In 14 (38.9\%) patients, $\mathrm{BH}$ persisted after the intervention on the head of the pancreas (in particular, in 8 patients with $\mathrm{BH}$ of the third degree and in 6 patients with $\mathrm{BH}$ of the second degree). For the correction of $\mathrm{BH}$, they were subjected to additional intervention on the CBD (Table II).

The use of the method of IOM BP allowed diagnosis of latent $\mathrm{BH}$ and assessment of the adequacy of the operation on the head of the pancreas to eliminate $\mathrm{BH}$. The sensitivity of this diagnostic method regarding $\mathrm{BH}$ was $97.3 \%$. 
Table II. Data on intraoperative monitoring of BP

\begin{tabular}{|c|c|c|c|c|c|c|c|c|}
\hline \multirow{3}{*}{$\begin{array}{l}\text { Degree of BH } \\
\text { at the beginning } \\
\text { of the surgery }\end{array}$} & \multicolumn{8}{|c|}{$\mathrm{BH}$ after the stage of surgery on the pancreatic head, $\mathrm{mm} \mathrm{Hg}$} \\
\hline & \multicolumn{2}{|c|}{ Norm } & \multicolumn{2}{|c|}{$1^{\text {st }}$ degree } & \multicolumn{2}{|c|}{$2^{\text {nd }}$ degree } & \multicolumn{2}{|c|}{$3^{\text {rd }}$ degree } \\
\hline & Mean & $\%$ & Mean & $\%$ & Mean & $\%$ & Mean & $\%$ \\
\hline $1(n=6)$ & 6 & 16.6 & - & - & - & - & - & - \\
\hline $2(n=22)$ & 16 & 44.4 & 4 & 11.1 & 2 & 5.6 & - & - \\
\hline $3(n=8)$ & - & - & 4 & 11.1 & 3 & 8.3 & 1 & 2.8 \\
\hline Total & 22 & 61.1 & 8 & 22.2 & 5 & 13.9 & 1 & 2.8 \\
\hline
\end{tabular}

A comprehensive study of $\mathrm{BH}$ became the basis for determining the sensitivity of instrumental methods for detecting $\mathrm{BH}$ in the case of $\mathrm{CP}$ (Figure 1). The data obtained indicate a high sensitivity of the methods of ERCP, MRCP, CT, and IOM BP.

The sensitivity of diagnostic methods in CP patients with $\mathrm{BH}$ with obstructive jaundice and without it was analysed separately. It was determined that the sensitivity of ultrasonography is significantly higher ( $p<$ $0.05)$ in patients with obstructive jaundice (87.1\%) than in patients without obstructive jaundice (42.5\%). The sensitivity of CT, ERCP, and MRCP does not depend on the presence of obstructive jaundice.

The research conducted allowed generalisation of the diagnostic criteria of $\mathrm{BH}$ when using different methods of examination, in particular:

- complaints: jaundice, itching, dark urine coloration, acholic stools;

- anamnesis: jaundice, surgeries on bile ducts;

- laboratory data: hyperbilirubinaemia, elevated levels of alkaline phosphatase;

- ultrasound examination: dilation of bile ducts $(8 \mathrm{~mm}$ and more), cysts of the head of the pancreas, concrements in bile ducts;

- CT: dilation of bile ducts, cysts of the head of the pancreas, concrements in bile ducts;

- ERCP: tubular stenosis of the bile ducts, its extent, suprastenotic dilation ( $8 \mathrm{~mm}$ and more), duration of the passage of contrast, concrements in bile ducts;

- MRCP: tubular stenosis of the CBD, its extent, suprastenotic dilation, concrements in bile ducts;

- intraoperative measurement of the diameter of the CBD: enlargement of the CBD of $8 \mathrm{~mm}$ or more;

- IOM BP: biliary pressure of more than $160 \mathrm{~mm}$ WG.

The research conducted became the basis for generalisation of diagnostic criteria of $\mathrm{BH}$ when using different methods of examination, and development of a diagnostic scheme for diagnosing $\mathrm{BH}$ in cases of $\mathrm{CP}$ (Figure 2).

Application of the developed scheme of $\mathrm{BH}$ diagnostics in cases of CP enabled an increase in the reliability

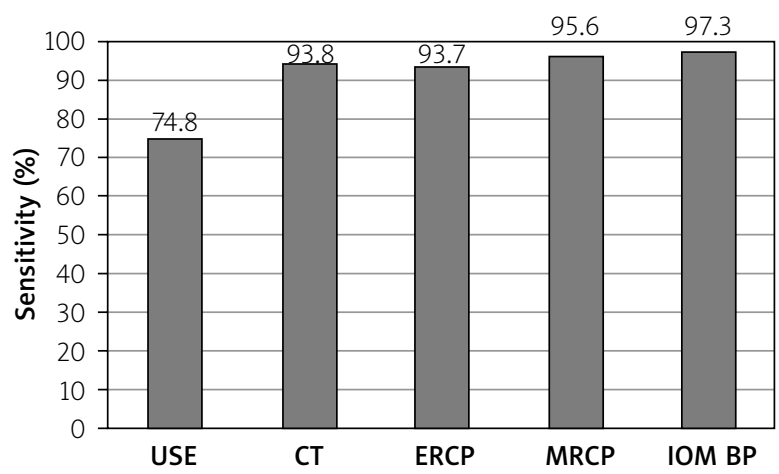

Figure 1. Sensitivity of instrumental methods of examination in diagnostics of $\mathrm{BH}$

of preoperative diagnostics to $95.6 \%$ and intraoperative diagnostics to $97.3 \%$, as well as allowing the choice of optimal method of surgical treatment.

The quality of life in the remote post-operative period was traced in 94 (57.6\%) patients: in 28 (77.7\%) patients of the first group and in $66(51.9 \%)$ patients of the second group. We observed 35 (37.2\%) patients ourselves, using clinical, laboratory and instrumental examinations, and filled out the SF-36 questionnaire. In $26(27.6 \%)$ patients, the results of surgical treatment were studied on the basis of medical documentation, which was maintained by surgeons and gastroenterologists in the patients' areas of residence. In $33(35.2 \%)$ patients, the remote postoperative results were assessed only on the basis of the SF-36 questionnaire.

Good results were indicated by the following: lack of clinical and laboratory signs and data received by instrumental methods that would prove the recurrence of $\mathrm{BH}$; absence of the abdominal pain syndrome, which existed before surgery; indicators of the exocrine and endocrine functions of the pancreas are almost the same as before surgery or even better; absence of CP complications, which served as indications for surgical intervention. Such results were obtained in 25 (89.2\%) patients of the first group and only 45 (68.2\%) patients of the sec- 


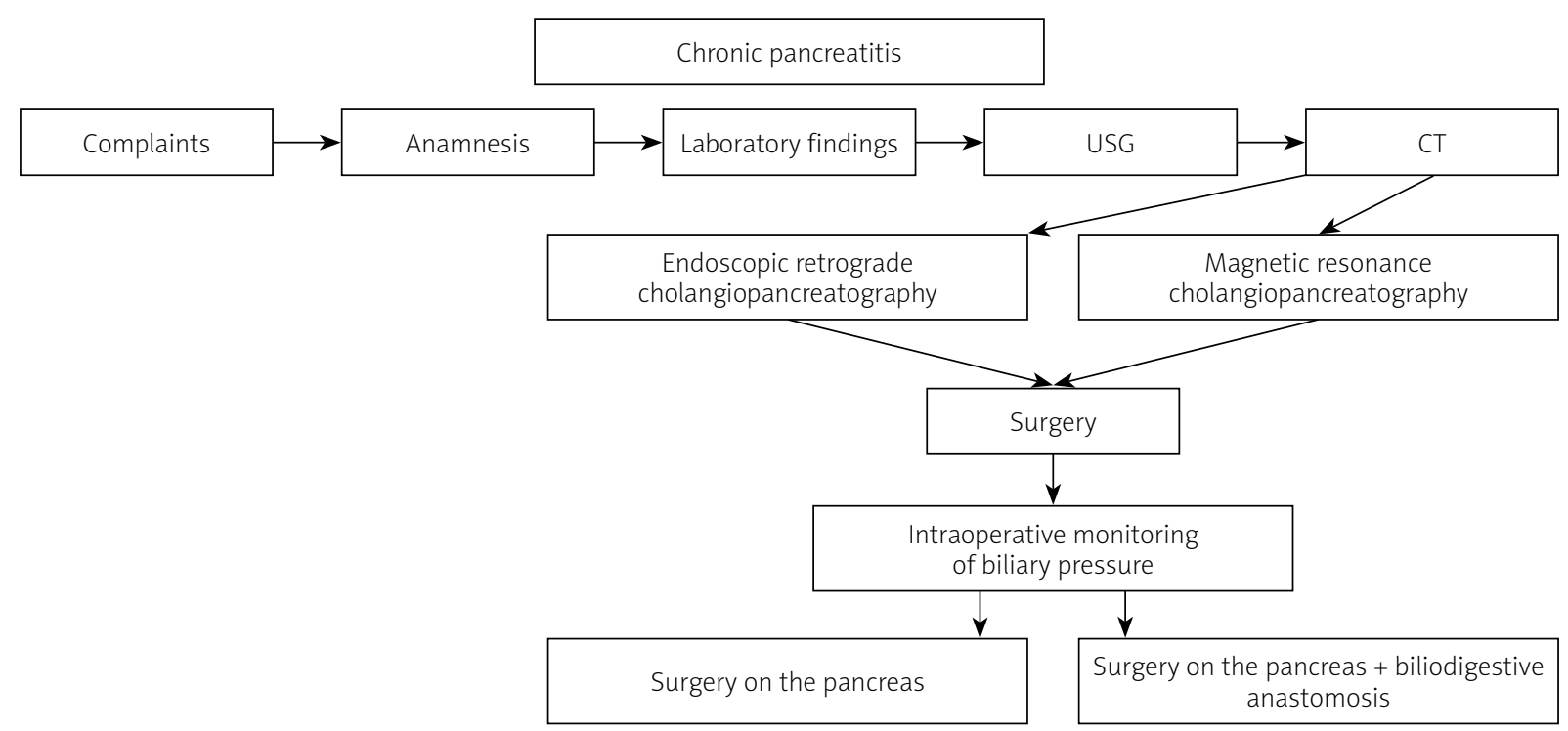

Figure 2. Scheme of diagnosing biliary hypertension in patients with chronic pancreatitis

ond group, despite the fact that the difference between these groups is not statistically significant $(p>0.05)$.

The following results were considered satisfactory: abdominal pain syndrome was either absent or significantly less intense and/or frequent than before the surgery; recurrent $\mathrm{BH}$, which did not require surgical intervention. Such results were obtained in 2 (7.1\%) patients of the first group and in 17 (25.7\%) patients of the second group (the difference between the groups is out of statistical control $-p>0.05$ ).

The results were considered unsatisfactory when patients suffered from abdominal pain and there current $\mathrm{BH}$ required surgical treatment. Such unsatisfactory results were found in $11(3.6 \%)$ patients of the first group and in $4(6.1 \%)$ patients of the second group.

The distribution of patients of both groups according to the remote postoperative results is presented in Table III.

Better remote postoperative results were obtained in patients, in whom BP was monitored. However, the difference of indicators is not statistically significant $\left(\chi^{2}=4.8, p=0.09\right)$.

The statistical analysis revealed the absence of any significant difference in the general remote results be- tween the two groups $\left(\chi^{2}=4.8, p=0.09\right)$. However, the research of the recurrence of $\mathrm{BH}$ in the remote postoperative period showed that patients who underwent IOMBP had no recurrence of $\mathrm{BH}$, while in the group, in which $\mathrm{BP}$ was not monitored, recurrent $\mathrm{BH}$ was diagnosed in 10 (15.1\%) patients. Thus, we found a significant difference between the two groups in terms of indicators characterising the development of recurrent $\mathrm{BH}$ in the remote postoperative period $\left(\chi^{2}=4.22, p=0.04\right)$. This allows us to recommend the developed method of IOMBP for broad use in clinical practice.

Statistical analysis of quality of life indicators of CP patients with $\mathrm{BH}$ in regard to the remote postoperative period showed that these data were much better than before surgery $(p<0.05)$. Nevertheless, no significant difference in the quality of life indicators between the two groups of patients was found $(p>0.05)$.

\section{Conclusions}

MRCP (95.6\%), ERCP (93.7\%), and CT (93.8\%) are the most sensitive of the main methods of diagnosing $\mathrm{BH}$ in cases of CP. ERCP is an invasive method, which is accompanied by postoperative complications in 14.6\% of patients; its use for diagnostics of biliary hyperten-

Table III. Assessment of the results of surgeries for $\mathrm{CP}$ with $\mathrm{BH}$ in the remote postoperative period

\begin{tabular}{|c|c|c|c|c|c|}
\hline \multirow[t]{2}{*}{ Results } & \multicolumn{2}{|c|}{$1^{\text {st }}$ group $(n=28)$} & \multicolumn{2}{|c|}{$2^{\text {nd }}$ group $(n=66)$} & \multirow[t]{2}{*}{$P$-value } \\
\hline & Mean & $\%$ & Mean & $\%$ & \\
\hline Good & 25 & 89.2 & 45 & 68.2 & $>0.05$ \\
\hline Satisfactory & 2 & 7.1 & 17 & 25.7 & $>0.05$ \\
\hline Unsatisfactory & 1 & 3.6 & 4 & 6.1 & $>0.05$ \\
\hline
\end{tabular}


sion should be limited. As non-invasive methods, MRCP and $\mathrm{CT}$ should be given preference in the algorithm of preoperative diagnostics. The use of the method of IOM BP allows an increase in the sensitivity of intraoperative diagnostics of $\mathrm{BH}$ to $97.3 \%$, helps to choose a method of surgical intervention, and significantly reduces the risk of relapse in the distant postoperative period by $15.1 \%\left(\chi^{2}=4.22, p=0.04\right)$.

\section{Conflict of interest}

The author declares no conflict of interest.

\section{References}

1. Prosolenko KA. Cure of patients with chronic pancreatitis according to the latest Ukrainian standards. Vestnik klub apankreatologov 2016; 2: 5-11.

2. Rusyn VI, Filip SS, Boldizhar OO, et al. Surgical treatment of chronic pancreatitis. Kharkiv Surgical School 2014; 2: 29-34.

3. Kryvoruchko IA, Boyko VV, Goncharova NN. Surgical treatment of chronic pancreatitis with regard to the classification of $\mathrm{M}$. Buchler et al. (2009). Suchasni medychni tekhnolohiyi 2011; 3-4: 195-8.

4. Kovalska I, Dronov O, Beregova T, et al. Changes of bile composition in patients with chronic pancreatitis. Pancreatology 2014; 14 (3 Suppl): S102.

5. Dobrov SD, Polyakevich AS, Blagitko EM, et al. Bile hypertension in patients with chronic pancreatitis. Annaly khirurgicheskoy gepatologii 2012; 4: 35.

6. Gagua AK, Vorobyov PY, Valkov KC, et al. Choose of the optimal volume of surgery in patients with chronic pancreatitis complicated by biliary hypertension. Materials of the XXIII International Congress on the Association of Hepatopancreatobiliary Surgeries of the CIS "Actual problems of hepatopancreatobiliary surgery". Minsk 2016; 230-1.

7. Sebastiano P, Molla FF. Pathophysiology of chronic damage. In: Acute and Chronic Pancreatitis: New concepts and evidence-based approaches. Testoni PA, Mariani A, Arcidiacono PG (eds). Edizioni Minerva Medica, Turin 2013; 63-9.

8. Kleeff J, Stöß C, Mayerleetal J. Evidence-based surgical treatments for chronic pancreatitis. Dtsch Arztebl Int 2016; 113: 489-96.

9. Grinevich VB, Maistrenko NA, Pryadko AS, et al. The problem of chronic pancreatitis from the position of a therapist and a surgeon. Meditsinskiy Akademicheskiy Zhurnal 2012; 12: 35-55.

10. Shuleyko AC, Vorobey AV, Grishin IN, et al. Biliary hypertension in patients with chronic pancreatitis. Materials of the Jubilee International Congress of Association of Surgeons-Hepatologists of CIS "Actual Problems of Surgical Hepatology”. Donetsk 2013; 301.

11. Drewes AM. Endoscopic management of complications of chronic pancreatitis. World J Gastroenterol 2013; 19: 7308-15.

12. Strate T, Taherpour Z, Bloechle C. Long-term follow-up of a randomized trial comparing the beger and frey procedures for patients suffering from chronic pancreatitis. Ann Surg 2005; 241: 591-8.
13. Maev IV, Ivashkin VT, Kaziulin AN, et al. Clinical recommendations for diagnosis and treatment of chronic pancreatitis. Moscow 2013; 42.

14. Johnstone M, Jackson R, Hannaetal T. The diagnosis of chronic pancreatitis: a systematic review. Pancreatology 2014; 14: 41.

15. Pat. 101713 Ukraine, IPC A 61B 17/00. Method of surgical treatment of chronic pancreatitis complicated with cholelithiasis. Pylypchuk VI, Kopchak VM, Shevchuk IM (Ukraine). No 2015 03315; stated 04/09/2015; published September 25, 2015 Bull No 18.

16. Pat. 109547 Ukraine, IPC A 61B 17/00. Method of measuring the pressure in the common bile duct. Pylypchuk VI, Kopchak VM, Shevchuk IM (Ukraine). No 2016 02279; stated March 10, 2016; published 08/25/2016 Bull No 16.

Received: 16.10 .2017

Accepted: 19.12 .2017 